\title{
Dynamics Of Collapsing Bubbles Near Walls
}

\author{
Sigrid Andreae ${ }^{1}$, Josef Ballmann ${ }^{1}$, Siegfried Müller ${ }^{2}$, and Alexander Voß ${ }^{2}$ \\ ${ }^{1}$ Lehr- und Forschungsgebiet für Mechanik der RWTH Aachen, Germany \\ sigrid.andreae@lufmech.rwth-aachen.de, ballmann@lufmech.rwth-aachen.de \\ 2 Institut für Geometrie und Praktische Mathematik, RWTH Aachen, Germany \\ mueller@igpm.rwth-aachen.de,voss@igpm.rwth-aachen.de
}

Summary. The influence of an adjacent wall on a collapsing bubble is investigated numerically. For this purpose, the two phase fluid is modeled by a single equation of state using the van der Waals equation of state and applying the Maxwell construction in the mixture region. Thereby the possibility of phase transition is included. The numerical simulations are performed using an adaptive finite volume scheme where the grid refinement strategy is based on multiresolution techniques.

\section{Introduction}

The formation and collapse of vapor bubbles in a liquid is called cavitation. Lord Rayleigh discovered that pressure waves emitted during the process of cavitation [8] may damage solids, e.g., marine screw propellers. However, up to now little is known about the mechanisms causing the damage of the solid.

Cavitation is induced by a pressure drop in the liquid below vapor pressure. Such a pressure decrease may occur due to local acceleration of the liquid flow caused by geometrical constraints, e.g., if the liquid flows through a narrow orifice or around an obstacle. In case, the pressure drops below vapor pressure, the liquid bursts and creates a free surface filled with gas and vapor - the bubble. Due to changes in the flow field, the pressure in the liquid may increase again causing the bubble to collapse. The collapse is accompanied by strong shock and rarefaction waves running into the bubble and the surrounding liquid. The shock wave focuses in the center of the bubble. This leads to extreme physical states in the interior. In addition, the shrinking of the bubble leads to a compression of the vapor. Both effects evoke an increase of pressure which bulges the bubble. Hereby, a dynamic oscillation process is initiated which finally leads to the collapse of the bubble. If the collapse takes place next to a solid, the pressure distribution becomes asymmetric and a liquid jet develops [7] which is either directed towards or away from the solid boundary. The direction of the jet depends on the elasticity of the solid and on a ratio determined by the initial distance between the boundary and the center of the bubble divided by the maximum extension of the bubble. This has been shown experimentally by Brujan et al. in [1], [2].

In order to investigate experimentally the dynamics of a bubble collapse, the bubble is produced by a laser pulse. Thereby the fluid is heated in the focus of the laser and forms a small, hot gas bubble at very high temperature. This experimental setup provides an exact positioning of the bubble. The processes taking place in the interior of the collapsing and oscillating bubble and the prediction of onset and extent of the cavitation damaging are still subject of theoretical and experimental research. However, small time and space scales as well as the complicated dynamics make an experimental approach difficult. Therefore numerical investigations are needed to reveal information about the wave dynamics in the fluid as well as the damaging of the solid. Of particular interest are pressure contours and velocity vectors in the liquid phase as requested in [2].

The primary objective of the present work is to provide an accurate prediction of all occuring wave phenomena. This concerns waves running into the liquid and the center of the bubble as well as the interaction of these waves with a neighboring solid. Normally, a collapsing gas bubble in a liquid is modeled as a two phase flow problem which means for each fluid a different equation of state is used. This can be done by using two meshes, whereby each mesh has to be updated in every step. Other techniques are tracking the boundary using a level-set or a volume-of-fluid method. Consequently, the phase boundary is modeled as a mathematically sharp boundary without a transition zone 
which is not physical. Actually, there is a region where vapor as well as liquid may be present, as will be discussed in Sec. 2.2. To overcome that problem a homogeneous description of both phases with one equation of state is used. The equation of state has to provide a physically correct representation of the transition zone as well as liquid and gas phase, see Sec. 2.1. Since cavitation may occur in injection nozzles, fuels should be included as possible fluids. Some of them belong to the group of retrograde and even BZT (Bethe-Zel'dovich-Thompson) fluids which make certain demands on the equation of state and possibly generate additional nonclassical wave effects. For details on BZT fluids and nonclassical waves see Thompson [9] and Cramer [3], respectively, as well as the literature cited there. BZT fluids will be discussed in Sec. 2.3.

The small time scales of the unsteady problem require the numerical scheme to be highly efficient regarding computational time and memory requirements. This is realized by a local grid refinement strategy. Furthermore, all physical relevant phenomena have to be reliably detected and adequately resolved. Moreover, the scheme has to be robust and must not exhibit numerical oscillations, e.g., pressure oscillations at the phase boundary. The details of the numerical scheme are presented in Sec. 3.

In Sec. 4 numerical results for a bubble collapse near a rigid wall are presented and the arising dynamic wave patterns are discussed.

\section{Physical Model}

For our investigations the fluid is modeled by the van der Waals equation of state. This gives a qualitatively good representation of the fluid in various important regions, namely the regimes of high compression during the collapse of the bubble, the area with negative nonlinearity near the critical point on account of BZT fluids, and the mixture region to allow for phase transition.

\subsection{Van der Waals Equation of State}

A vital assumption of the ideal gas model is that the particles are non-interacting masspoints, i.e., an appreciable force acts on them only during a collision. Furthermore, the volume of the particles is negligible compared to the total volume occupied by the gas. This is no longer valid if the density is so high that the distance between two particles is of the order of their interaction diameter. Such extreme densities can be reached during the collapse of the bubble. In this case, the range of validity of the ideal gas is exceeded which is investigated in [4].

The van der Waals equation of state is an extension of the ideal gas model with two material parameters $a$ and $b$ to take into account the attraction of particles and the reduction of free volume. The thermal equation of state is determined by

$$
p(v, T)=\frac{R T}{v-b}-\frac{a}{v^{2}}
$$

with pressure $p$, temperature $T$, specific volume $v$, specific gas constant $R$, internal pressure $a / v^{2}$ and covolume $b$ covolume. The internal pressure is subtracted from the pressure to take into account that the attraction of particles diminishes the pressure. The covolume $b$ reduces the volume $v$ to the available free volume $v-b$. The caloric equation of state reads

$$
e(v, T)=e_{0}+c_{v}(v, T) T-\frac{a}{v}
$$

where $c_{v}$ denotes the heat capacity at constant volume and $e$ the internal energy. 


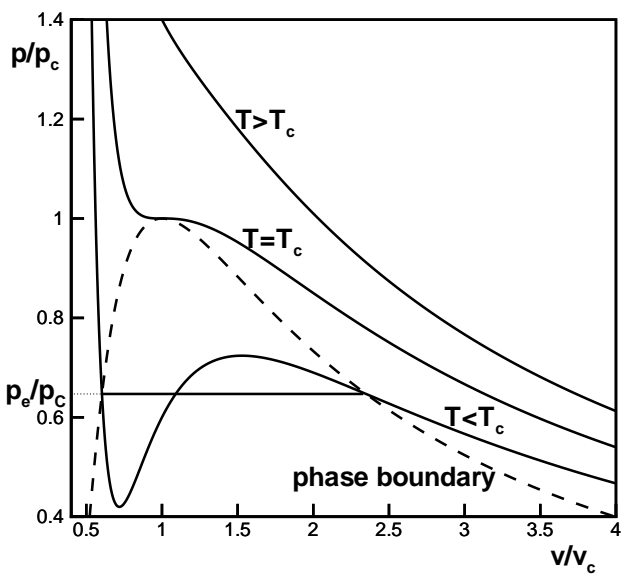

Fig. 1. Isotherms in $p$-v-diagram

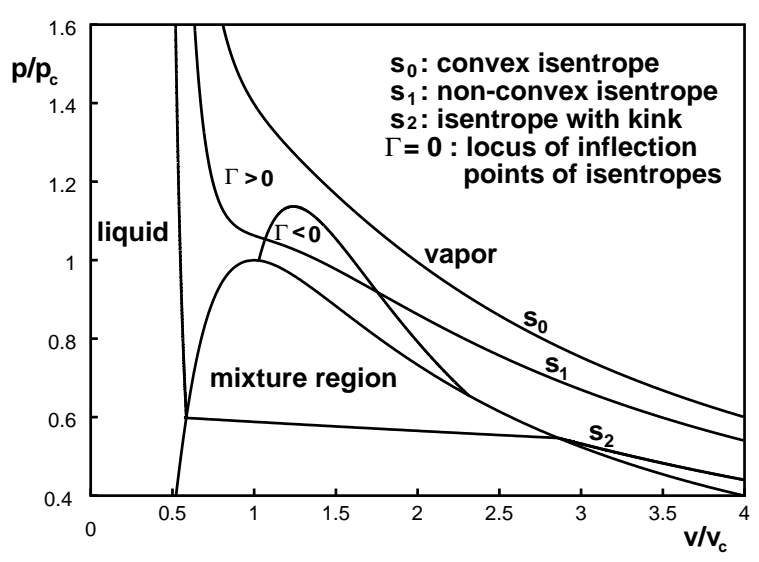

Fig. 2. Isentropes in $p$ - $v$-diagram

\subsection{Mixture Region}

Since we want to apply the van der Waals equation of state to gases and liquids as well, there is a need for describing of what happens in the region of phase transition. In the $p$ - $v$ diagram in Fig. 1, three isotherms are plotted corresponding to (i) a temperature above the critical temperature $\left(T_{c}\right)$, (ii) the critical temperature (the critical isotherm) and (iii) a temperature below $T_{c}$. In the mixture region, liquid and gas are present in a continuous changing fraction where the fraction of liquid rises when the volume decreases. In the mixture area, the van der Waals equation shows an unphysical inclination in the $p$ - $v$-diagram. Considering an isotherm crossing the mixture region, we observe two local extrema called spinodal points. They correspond to the endpoints of supersaturated vapor (maximum) and overexpanded liquid (minimum), respectively. Between these two points the derivative $\left.\frac{\partial p}{\partial v}\right|_{T}$ is positive and hence physically excluded. In order to provide physically meaningful data, we calculate the equilibrium pressure $p_{\mathrm{e}}$, see Fig. 1, as a function of volume and temperature using the Maxwell construction. For details on the implementation see [10], [11].

\subsection{BZT Fluids}

BZT fluids are high molecular hydrocarbons or -fluorids. They are a subgroup of retrograde fluids which exhibit a characteristic overhang of the saturation curve in the T-s-diagram. Fig. 3 and 4 show the difference between a generic and a retrograde fluid, respectively. The $T$-s-diagram for the generic fluid shows that the fluid has to be cooled for an equilibrium condensation [9]. However, the overhang in the $T$-s-diagram for the retrograde fluid implies that the retrograde fluid has to be heated for an equilibrium condensation. For the BZT fluid the curvature of the saturation curve

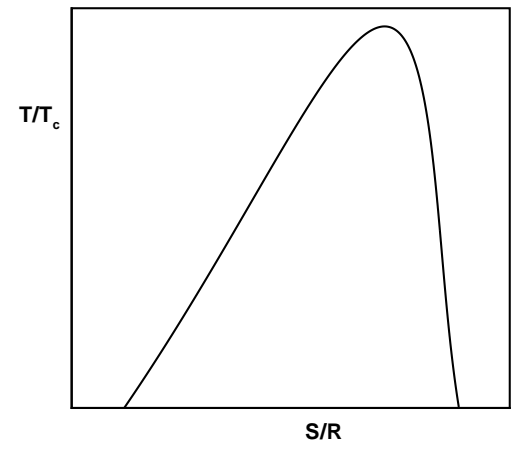

Fig. 3. $T$-s-diagram for $\mathrm{C}_{2} \mathrm{H}_{4}$

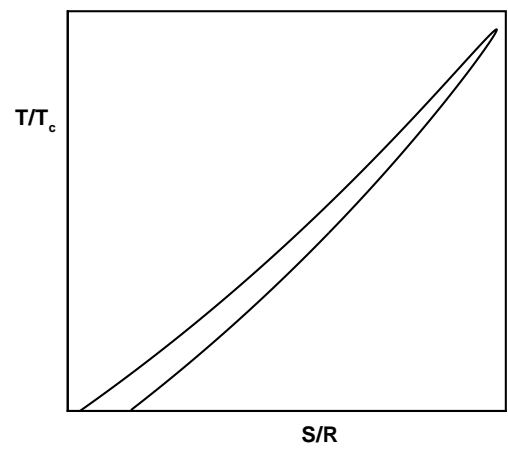

Fig. 4. $T$-s-diagram for $\mathrm{PP}_{10}$ 
is so intense that it leads to a concave bending of the isentropes near the critical point in the $p-v$ plane, see Fig. 2. This is in contrast to the behavior of the most common fluids which isentropes are convex. A measure for the bending is the fundamental derivative of gas dynamics

$$
\Gamma=\frac{v^{3}}{2 c^{2}} \frac{\partial^{2} p}{\partial v^{2}}
$$

which becomes negative in the respective region. Here, $c$ denotes the speed of sound. The gasdynamic peculiarity of the $\Gamma<0$-region implies the possibility of compression waves and expansion shocks [3]. In the later case, the pressure jump has to be positive to fulfill the entropy condition. The chosen van der Waals equation of state provides a good representation of the effects in the region of negative $\Gamma$. For more details on BZT fluids see Thompson [9].

\section{Governing Equations and Method of Solution}

The fluid flow is modeled by the time-dependent 2D Euler equations for compressible fluids.

$$
\begin{aligned}
& \frac{\partial}{\partial t} \int_{V} \mathbf{U} \mathbf{d} \mathbf{V}+\oint_{\partial \mathbf{V}}\left(\mathbf{F}^{\mathbf{c}}-\mathbf{F}^{\mathbf{v}}\right) \cdot \mathbf{n} \mathbf{d} \mathbf{S}=\mathbf{0} \text { with } \\
& \mathbf{U}=\left(\begin{array}{c}
\rho \\
\rho \mathbf{u} \\
\rho E
\end{array}\right), \quad \quad \mathbf{F}^{\mathbf{c}}=\left(\begin{array}{c}
\rho \mathbf{u} \\
\rho \mathbf{u} \circ \mathbf{u}+\mathbf{p} \mathbf{1} \\
\mathbf{u}(\rho \mathbf{E}+\mathbf{p})
\end{array}\right), \quad \quad \mathbf{F}^{\mathbf{v}}=\left(\begin{array}{c}
\mathbf{0} \\
\boldsymbol{\tau} \\
\mathbf{u} \cdot \boldsymbol{\tau}-\mathbf{q}
\end{array}\right) .
\end{aligned}
$$

$\mathbf{U}$ is the array of the mean conserved quantities: density, momentum, and specific total energy. $\tau$ is the viscous strain tensor and $\mathbf{q}$ the heat flux vector. The quantity $V$ denotes a time-independent control volume with the boundary $\partial V$ and the outer normal $\mathbf{n}$. The flux array is divided into its convective $\left(\mathbf{F}^{\mathbf{c}}\right)$ and diffusive $\left(\mathbf{F}^{\mathbf{v}}\right)$ part.

The transport equations are solved using an explicit finite volume method. For the convective terms a Godunov-type upwind scheme is applied. To improve the spatial accuracy we apply a higher order ENO reconstruction. The efficiency of the finite volume method is significantly improved by an adaptive grid refinement strategy which is based on multiresolution techniques that have been recently developed and investigated in [6].

Since BZT fluids exhibit wave phenomena different from common fluids, these have to be properly addressed by the numerical algorithms. For this purpose, the approximate Riemann solver due to Roe has to be modified with regard to a general equation of state, i.e., $p=p(v, e)$. The derivation is analogously to the approach of Liu and Vinokur, see [5]. As shown in [11] the numerical method is able to resolve expansion shocks as well as wave splitting phenomena.

\section{Numerical Results}

The focus of the current work is to verify the ability of the scheme to handle steep density gradients and to resolve the dynamic wave patterns accurately. Therefore, as a first step a generic fluid with initial conditions away from the phase transition is considered for the two-dimensional case. However, the initial conditions exhibit data differences which may be comparable to a phase boundary. Where a hot bubble at low pressure is enclosed by cold air at high pressure, see Fig. 5 . The fluid is modeled by the van der Waals equation. Here we still assume constant heat capacity $c_{v}, \gamma=1.4$ and $R=287 \mathrm{~J} / \mathrm{mol} / \mathrm{K}$. The initial conditions correspond to a Riemann problem where three types of waves occur: an inward running compression shock, an outward running rarefaction wave and a contact discontinuity. At time $t=0.01640 \mathrm{~ms}$ the fastest wave - the inward running shock - has reached the center of the bubble and is reflected, see Fig. 7. The pressure in the focus exceeds $40 \times 10^{6} \mathrm{~N} / \mathrm{m}^{2}$. The outward running rarefaction is visible in the pressure as well as in the density. Whereas the contact discontinuity is only seen in the density (see Fig. 6 at $0.068 \mathrm{~m}$ ). 


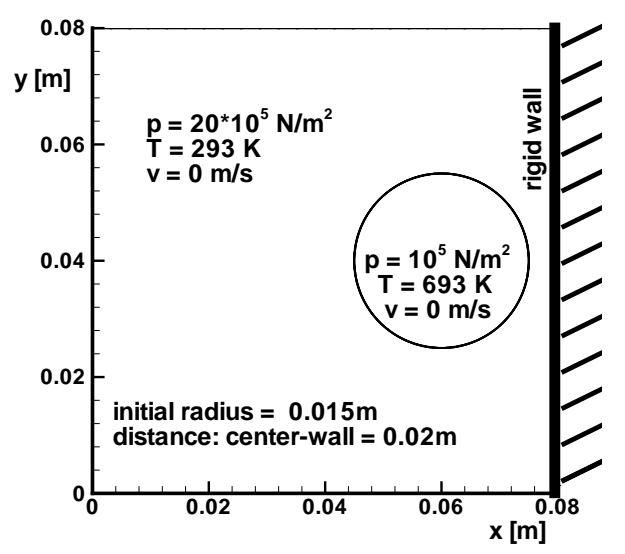

Fig. 5. Initial conditions and computational domain of the planar problem

It just reaches the solid wall. After the reflection in the center, the shock wave runs outward and passes the contact discontinuity. Hereby, the shock is partly transmitted and partly reflected. The reflected part runs again into the center, is reflected and passes the contact discontinuity again. The rarefaction wave is reflected at the solid wall as rarefaction. The corresponding doubling of the amplitude produces a region of low pressure which increases the shock pressure ratio and, therfore, accelerates the shock wave towards the wall, see Fig. 8 and 9, where it is reflected, see Fig. 11. The reflection area extends along the wall developing a Mach stem, see Fig. 12, at time $t=0.14925 \mathrm{~ms}$. In the density contours the slip line, a contact discontinuity originating at the triple point of the Mach stem, is clearly visible. The discontinuity separates two regions of different values of entropy that are caused in the material passing through shocks of different strength. The Mach stem The pressure distribution on the solid (right) and the density contours (left) are presented to display the loading jump on the solid evoked by the Mach stem (visible at $y=0.074 m$ and $y=0.006 m$ ). To identify the remainder of the bubble, Fig. 13 shows the pressure and density distribution on the symmetry line at $y=0.04$ for the same time. The contact discontinuity is characterized by a jump in density and nearly constant pressure between $x=0.062 \mathrm{~m}$ and $x=0.067 \mathrm{~m}$.

As mentioned earlier, the initial conditions determine whether the bubble is repelled from or attracted towards the wall. In addition, the surrounding fluid is accelerated such that a jet of cold gas occurs in wall direction or opposite. In this configuration cold gas flows away from the wall, repells the hot bubble from the solid and finally splits it. This is observable by the velocity in $x$-direction and the integral curves of the velocity direction field which is marked by arrow-lines in Fig. 14 at time $t=0.70417 \mathrm{~ms}$. In the experiments [1] for rigid wall a jet directed versus the wall was observed. The opposite in this computation is caused by the discrepancy of the initial conditions concerning the velocity field on both sides of the bubble surface.

\section{Conclusion and Future Work}

The numerical method is able to handle the small time scales occuring during the collapse of a bubble due to the grid refinement strategy. Besides, it is quite stable even at steep density gradients. The interaction of the shock wave and the rarefaction wave as well as the reflection of both waves on the rigid wall are well resolved. In particular, the development of the Mach stem is traced accurately. The contact discontinuity exhibits instabilities which are due to the Cartesian grid.

In future work, the position of the bubble will be varied in order to compute the prediction of Brujan et al. [1] that the direction of the jet depends on the distance between boundary and bubble. Furthermore, initial states near the critical point will be considered to investigate the influence of retrograde behavior and effects caused by phase transition. 


\section{Acknowledgement}

This work is financially supported by the DFG Priority Research Program "Analysis and Numerics for Conservation Laws".

\section{References}

1. Brujan, E.-A., Nahen, K., Schmidt, P., Vogel, A. (2001): Dynamics of laser-induced cavitation bubbles near an elastic boundary. J. Fluid Mech., 433, 251-281

2. Brujan, E.-A., Nahen, K., Schmidt, P., Vogel, A. (2001): Dynamics of laser-induced cavitation bubbles near elastic boundaries: influence of the elastic modulus. J. Fluid Mech., 433, 283-314

3. Cramer, M. S. (1991): Nonclassical dynamics of classical waves. In: Kluwick, A. (ed) Nonlinear Waves in Real Fluids. Springer Wien New York

4. Hanke, M., Ballmann, J. (1998): Strong changes of state in collapsing bubbles. ZAMM, 78, 453-453

5. Liu Y., Vinokur, M. (1989): Nonequilibrium flow computations. I. An analysis of numerical formulations of conservation laws. J. Comp. Phys., 83, 373-397

6. Müller, S., (2002): Adaptive Multiscale Schemes for Conservation Laws. Accepted for publication in Lecture Notes on Computational Sciences and Engineering. Springer

7. Philipp, A., Lauterborn, W. (1998): Cavitation erosion by single laser-produced bubbles. J. Fluid Mech., 361, $75-116$

8. Lord Rayleigh (1917): On the pressure developed in a liquid during the collapse of a spherical cavity. Phil. Mag., 34, 94-98

9. Thompson, P. A. (1991): Liquid-vapor adiabatic phase changes and related phenomena. In: Kluwick, A. (ed) Nonlinear Waves in Real Fluids. Springer Wien New York

10. Voß, A. (2002): A Numerical Library for Fluids and Convex and Nonconvex EOS Including Phase Transition. IGPM-report, RWTH Aachen, in preparation

11. Voß, A. : Flows in Fluids with Nonconvex Equation of State. PhD Thesis, RWTH Aachen, in preparation 


\section{Figures}

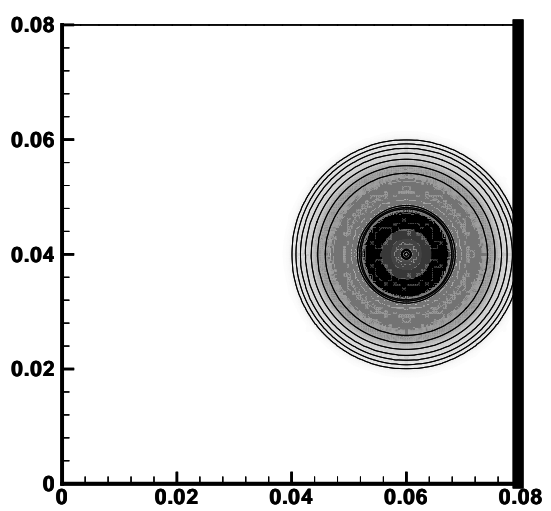

$\left[\mathbf{p g} / \mathbf{m}^{3}\right]$

$\begin{array}{lllllll}1.78 & 5.50 & 9.23 & 12.95 & 16.68 & 20.40 & 24.12\end{array}$

Fig. 6. $t=0.01640 \mathrm{~ms}$

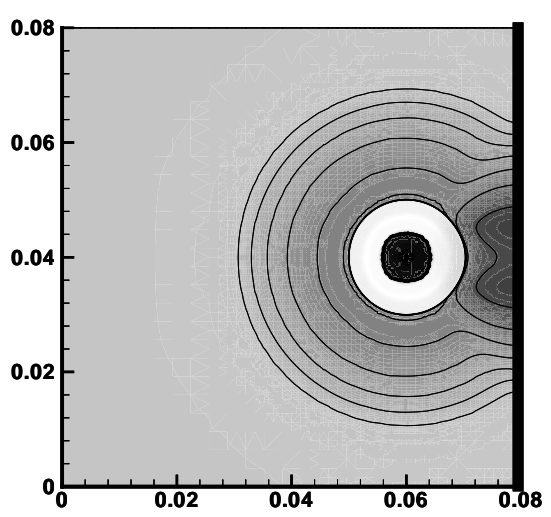

$\left[\mathbf{k g} / \mathbf{m}^{3}\right]$

$\begin{array}{lllllll}5.51 & 10.37 & 15.23 & 20.09 & 24.96 & 29.82 & 34.68\end{array}$

Fig. 8. $t=0.04851 \mathrm{~ms}$

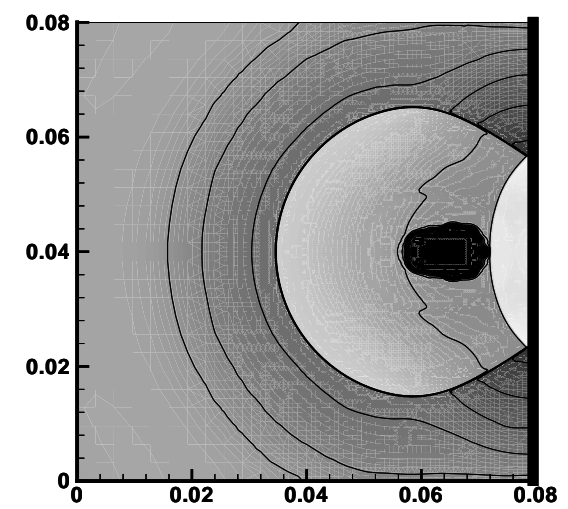

$\left[\mathbf{k g} / \mathbf{m}^{3}\right]$

$\begin{array}{lllllll}5.51 & 10.37 & 15.23 & 20.09 & 24.96 & 29.82 & 34.68\end{array}$

Fig. 10. $t=0.10150 \mathrm{~ms}$

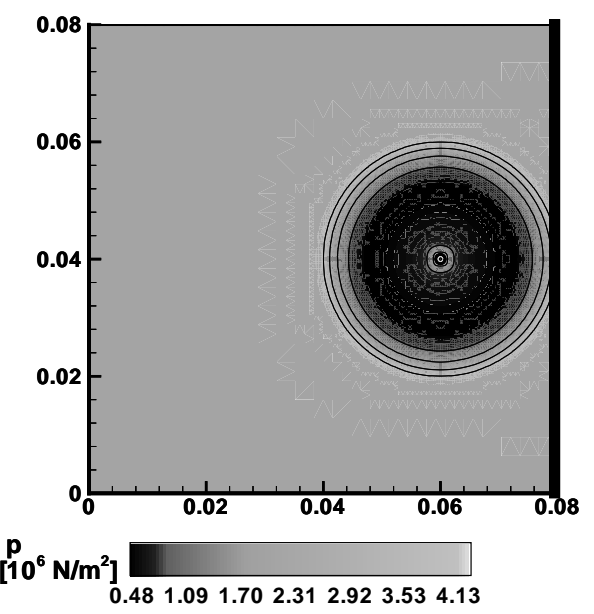

Fig. 7. $t=0.01640 \mathrm{~ms}$

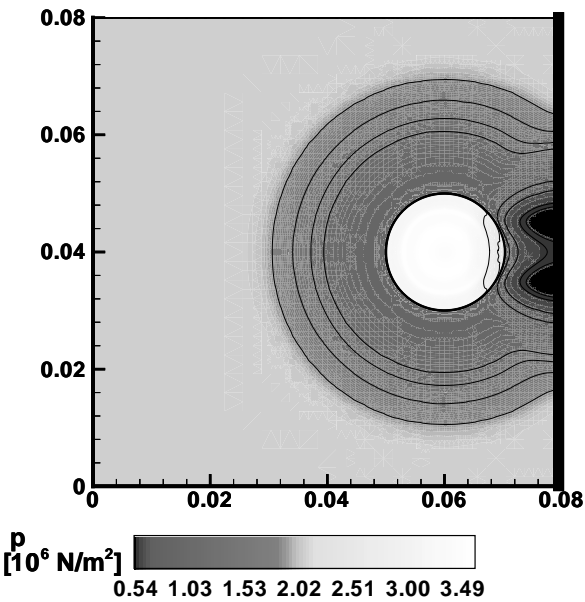

Fig. 9. $t=0.04851 \mathrm{~ms}$

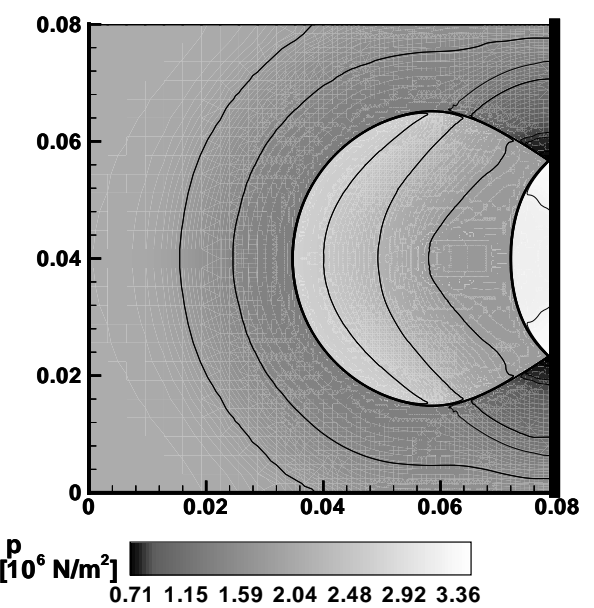

Fig. 11. $t=0.10150 \mathrm{~ms}$ 
Sigrid Andreae, Josef Ballmann, Siegfried Müller, and Alexander Voß

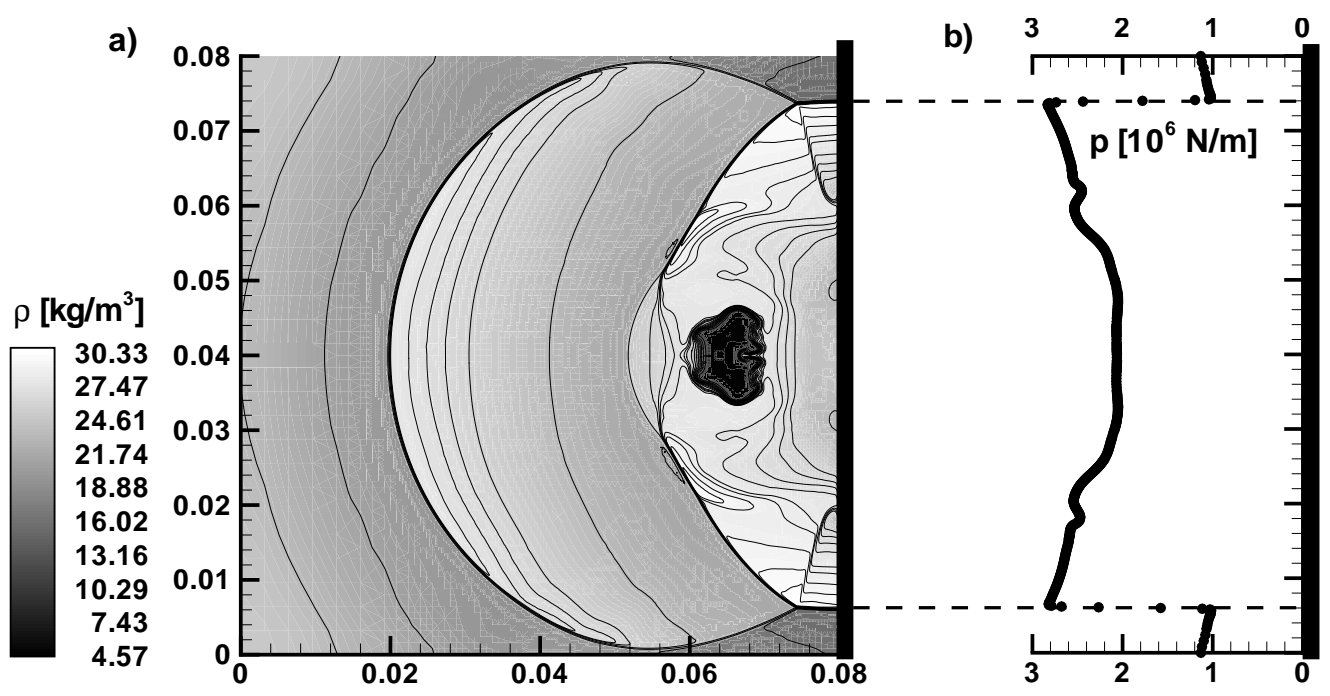

Fig. 12. $t=0.14925 m s$ : a) density contours, b) pressure distribution at wall

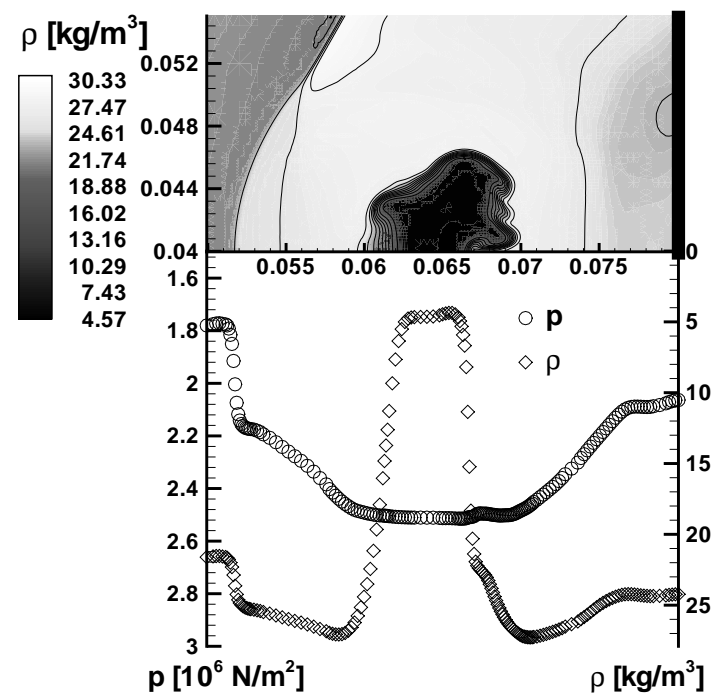

Fig. 13. $t=0.14925 \mathrm{~ms}$ :

density contours (top), density and pressure at $y=0.04 m$ (bottom)

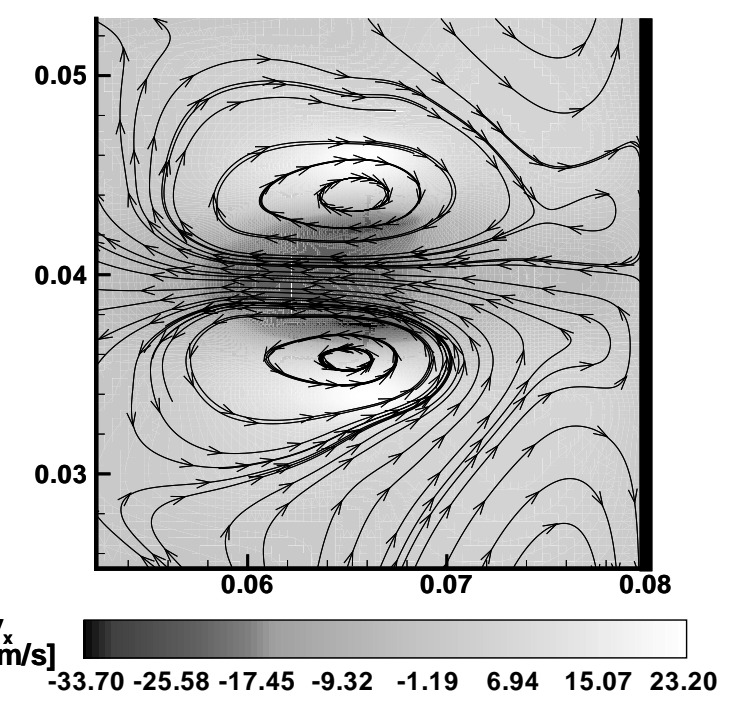

Fig. 14. $t=0.70417 m s$ :

velocity component $v_{x}$ (greyscale),

integral curves of velocity direction field (arrows) 